\title{
ERROR BOUNDS FOR GENERAL MIXED QUASIVARIATIONAL INEQUALITIES
}

\author{
MUHAMMAD ASLAM NOOR AND KHALIDA INAYAT NOOR
}

Received 8 August 2004 and in revised form 19 December 2004

It is well known that mixed quasivariational inequalities are equivalent to the implicit fixed-point problems. We use this alternative equivalent formulation to suggest and consider some merit functions for general mixed quasivariational inequalities. We use these merit functions to obtain error bounds for the solution under some mild conditions. Some special cases are also discussed.

\section{Introduction}

Variational inequalities introduced by Stampacchia [25] in the early sixties have been generalized and extended in various directions using innovative techniques. A useful and significant generalization of variational inequalities is called the mixed quasivariational inequality involving the nonlinear bifunction which enables us to study the free, moving, unilateral, and equilibrium problems arising in elasticity, fluid flow through porous media, finance, economics, transportation, circuit, and structural analysis in a unified framework; see $[1,2,3,4,5,6,7,8,9,10,11,12,13,14,15,16,17,18,19,20,21,22$, $23,24,25,26]$. As a result of interaction between different branches of mathematical and engineering sciences, we now have a variety of techniques including the projection methods and their variant forms, auxiliary principle, resolvent equations to suggest and analyze various iterative algorithms for solving variational inequalities, and related optimization problems. It is well known that the projection method and its variant forms cannot be extended for mixed quasivariational inequalities due to the presence of the bifunction. However, if the bifunction is a proper, convex, and lower semicontinuous function with respect to the first argument, then it has been shown (see [13]) that the mixed quasivariational inequalities are equivalent to the fixed-point problem. This alternative equivalent formulation has been used to suggest and analyze some iterative methods for solving mixed quasivariational inequalities. Using this alternative equivalence, we define the natural residue vector, which is also known as the merit function. In recent years, much attention has been given to construct and investigate some regularized and $D$-merit functions associated with classical variational inequalities. These merit functions play an important part in developing several iterative methods for solving variational inequalities 
and related optimization problems; see $[4,19,20,21,22,23,24,26]$. On the other hand, there are no such merit functions for mixed quasivariational inequalities. In this paper, we consider and investigate some merit functions for mixed quasivariational inequalities and use these merit functions to obtain error bounds for the solution of mixed quasivariational inequalities. As special cases, we obtain some new and previously known results for variational inequalities and related problems. Thus the results obtained in this paper can be viewed as an extension and refinement of previously known results.

\section{Formulations and basic facts}

Let $H$ be a real Hilbert space whose inner product and norm are denoted by $\langle\cdot, \cdot\rangle$ and $\|\cdot\|$, respectively. Let $K$ be a closed convex set in $H$ and $T, g: H \rightarrow H$ nonlinear operators. Let $\varphi(\cdot, \cdot): H \times H \rightarrow R \cup\{+\infty\}$ be a continuous bifunction with respect to both arguments. We consider the problem of finding $u \in H: g(u) \in H$ such that

$$
\langle T u, g(v)-g(u)\rangle+\varphi(g(v), g(u))-\varphi(g(u), g(u)) \geq 0, \quad \forall v \in H: g(v) \in H,
$$

which is called the general mixed quasivariational inequality and has been studied extensively in recent years; see $[14,17]$.

If the bifunction $\varphi(\cdot, \cdot)$ is proper, convex, and lower semicontinuous with respect to the first argument, then problem (2.1) is equivalent to finding $u \in H: g(u) \in H$ such that

$$
0 \in T u+\partial \varphi(g(u), g(u))
$$

which is known as finding a zero-sum of two (more) maximal monotone operators and has been studied extensively in recent years.

For $g \equiv I$, the identity operator, problem (2.1) reduces to the following problem. Find $u \in H$ such that

$$
\langle T u, v-u\rangle+\varphi(v, u)-\varphi(u, u) \geq 0, \quad \forall v \in H .
$$

Problem (2.3) is called the mixed quasivariational inequality; see $[1,2,3,8,11,13,14,15$, 17].

If $\varphi(u, v)=\varphi(v)$, for all $v \in H$, then problem (2.1) is equivalent to finding $u \in H$ : $g(u) \in H$ such that

$$
\langle T u, g(v)-g(u)\rangle+\varphi(g(v))-\varphi(g(u)) \geq 0, \quad \forall v \in H: g(v) \in H,
$$

which is called the general mixed variational inequality.

If the bifunction $\varphi(\cdot)$ is the indicator function of a closed and convex set $K$ in $H$, that is,

$$
\varphi(u)= \begin{cases}0 & \text { if } u \in K, \\ +\infty & \text { otherwise }\end{cases}
$$

then problem (2.4) is equivalent to finding $u \in H, g(u) \in K$ such that

$$
\langle T u, g(v)-g(u)\rangle \geq 0, \quad \forall g(v) \in K,
$$


which is known as the general variational inequality introduced and studied by Noor [10] in 1988. It turned out that a wide class of nonsymmetric and odd-order free, moving and equilibrium problems arising in finance, economics, transportation, elasticity, telecommunication network, optimization, and operations research can be studied in the unified and general framework of problems (2.1)-(2.6). For $g \equiv I$, the identity operator, we obtained the corresponding classical variational inequality problems; see $[1,2,3,4,5,6,7$, $8,9,10,11,13,14,15,16,17,18,19,20,21,22,23,24,25,26]$.

We also need the following well-known results and concepts.

Definition 2.1. The operator $T: H \rightarrow H$ is said to be

(a) strongly $g$-monotone if and only if there exists a constant $\alpha>0$ such that

$$
\langle T u-T v, g(u)-g(v)\rangle \geq \alpha\|g(u)-g(v)\|^{2}, \quad \forall u, v \in H
$$

(b) $g$-monotone if and only if

$$
\langle T u-T v, g(u)-g(v)\rangle \geq 0, \quad \forall u, v \in H ;
$$

(c) $g$-Lipschitz continuous if there exists a constant $\beta>0$ such that

$$
\|T u-T v\| \leq \beta\|g(u)-g(v)\|, \quad \forall u, v \in H
$$

(d) hemicontinuous if for all $u, v \in H$, the mapping $t \in[0, t]$ implies that

$$
\langle T(u+t(v-u)), v\rangle
$$

is continuous.

From (a) and (c), we have $\alpha \leq \beta$. For $g=I$, the identity operator, Definition 2.1 reduces to the well-known definition of strongly monotone and Lipschitz continuity of $T$.

Remark 2.2. We would like to point out that if the operator $T$ is strongly monotone with a constant $\alpha>0$, then

$$
\alpha\|u-v\|^{2} \leq\langle T u-T v, u-v\rangle \leq\|T u-T v\|\|u-v\|
$$

implies that

$$
\|T u-T v\| \geq \alpha\|u-v\|, \quad \forall u, v \in H .
$$

In this case, we say that the operator $T$ is strong nonexpansion with a constant $\alpha>0$. Note that the strong monotonicity implies expansioncivity, but not conversely. It is clear that if the operator $T$ is strongly $g$-monotone and $g$ is strongly nonexpansion, then

$$
\langle T u-T v, g(u)-g(v)\rangle \geq \alpha\|g(u)-g(v)\|^{2} \geq \alpha\|u-v\|^{2}, \quad \forall u, v \in H .
$$

Definition 2.3. The bifunction $\varphi(\cdot, \cdot)$ is said to be skew-symmetric if and only if

$$
\varphi(u, u)-\varphi(u, v)-\varphi(v, u)+\varphi(v, v) \geq 0, \quad \forall u, v \in H .
$$


1088 Error bounds for quasivariational inequalities

Clearly, if the skew-symmetric bifunction $\varphi(\cdot, \cdot)$ is linear in both arguments, then $\varphi(u$, $u) \geq 0$, for all $u \in H$. In fact,

$$
\varphi(u, u)-\varphi(u, v)-\varphi(v, u)+\varphi(v, v)=\varphi(u-v, u-v) \geq 0, \quad \forall u, v \in H .
$$

Definition 2.4 (see [1]). Let $A$ be a maximal monotone operator, then the resolvent operator associated with $A$ is defined as

$$
J_{A}(u)=(I+\rho A)^{-1}(u), \quad \forall u \in H,
$$

where $\rho>0$ is a constant and $I$ is the identity operator.

Remark 2.5. It is well known that the subdifferential $\partial \varphi(\cdot, \cdot)$ of a convex, proper, and lower semicontinuous function $\varphi(\cdot, \cdot): H \times H \rightarrow R \cup\{+\infty\}$ is a maximal monotone with respect to the first argument, its resolvent is defined by

$$
J_{\varphi(u)}=(I+\rho \partial \varphi(\cdot, u))^{-1} \equiv(I+\rho \partial \varphi(u))^{-1},
$$

where $\partial \varphi(u) \equiv \partial \varphi(\cdot, u)$, unless otherwise specified.

The resolvent operator $J_{\varphi(g(u))}$ has the following characterization.

Lemma 2.6. For a given $u \in H: g(u) \in H, z \in H: g(z) \in H$ satisfies the inequality

$$
\langle g(u)-g(z), g(v)-g(u)\rangle+\rho \varphi(g(v), g(u))-\rho \varphi(g(u), g(u)) \geq 0, \quad \forall v \in H: g(v) \in H,
$$

if and only if

$$
g(u)=J_{\varphi(g(u))} g(z),
$$

where $J_{\varphi(g(u))}=(I+\rho \partial \varphi(\cdot, g(u)))^{-1}$ is the resolvent operator and $\rho>0$ is a constant.

Proof. Clearly,

$$
\begin{aligned}
(2.18) & \Longleftrightarrow g(z)-g(u) \in \rho \partial \varphi(g(u), g(u)) \\
& \Longleftrightarrow g(z) \in g(u)+\rho \partial \varphi(g(u), g(u)) \equiv(I+\rho \partial \varphi(\cdot, g(u)))(g(u)) \\
& \Longleftrightarrow g(u)=(I+\rho \partial \varphi(\cdot, g(u)))^{-1} g(z) \equiv J_{\varphi(g(u))} g(z),
\end{aligned}
$$

the required result.

Lemma 2.7. Let the operator $T$ be $g$-monotone and hemicontinuous and let the operator $g$ be convex. If the bifunction $\varphi(\cdot, \cdot)$ is convex in the first argument, then problem (2.1) is equivalent to finding $u \in H$ such that

$$
\langle T v, g(v)-g(u)\rangle+\varphi(g(v), g(u))-\varphi(g(u), g(u)) \geq 0, \quad \forall v \in H .
$$

Proof. Let $u \in H$ be a solution of (2.1). Then

$$
\langle T u, g(v)-g(u)\rangle+\varphi(g(v), g(u))-\varphi(g(u), g(u)) \geq 0, \quad \forall v \in H,
$$


which implies, using the $g$-monotonicity of $T$,

$$
\langle T v, g(v)-g(u)\rangle+\varphi(g(v), g(u))-\varphi(g(u), g(u)) \geq 0, \quad \forall v \in H .
$$

Conversely, let $u \in H$ be such that (2.21) holds. For $t \in[0,1], u, v \in H, v_{t}=u+t(v-u) \in$ $H$. Taking $v=v_{t}$ in (2.21), we have

$$
\begin{aligned}
0 & \leq t\left\langle T v_{t}, g(v)-g(u)\right\rangle+\varphi\left(g\left(v_{t}\right), u\right)-\varphi(u, u) \\
& \leq t\left\langle T v_{t}, g(v)-g(u)\right\rangle+t\{\varphi(g(v), g(u))-\varphi(g(u), g(u))\},
\end{aligned}
$$

since $g$ is convex and $\varphi(\cdot, \cdot)$ is also convex with respect to the first argument.

Dividing the above inequality by $t$ and letting $t \rightarrow 0$, we have

$$
\langle T u, g(v)-g(u)\rangle+\varphi(g(v), g(u))-\varphi(g(u), g(u)) \geq 0, \quad \forall v \in H,
$$

the required (2.1).

Remark 2.8. Inequality of type (2.21) is called the dual general mixed quasivariational inequality. From Lemma 2.7, it is clear that the solution sets of both problems (2.1) and (2.21) are equivalent. Lemma 2.7 plays an important part in the approximation of the variational inequalities. Lemma 2.7 can be viewed as a natural generalization of Minty's lemma (see [9]).

We now study those conditions under which the mixed quasivariational inequality (2.1) has a unique solution, which is the main motivation of our next result.

Theorem 2.9. Let $T$ be a strongly g-monotone with constant $\alpha>0$ and g-Lipschitz continuous operator with constant $\beta>0$. Let $g$ be an injective operator. If the bifunction $\varphi(\cdot, \cdot)$ is skew-symmetric and $0<\rho<2 \alpha / \beta^{2}$, then the general mixed quasivariational inequality (2.1) has a unique solution.

Proof. (a) Uniqueness. Let $u_{1} \neq u_{2} \in H$ be two solutions of (2.1). Then, we have

$$
\begin{array}{ll}
\left\langle T u_{1}, g(v)-g\left(u_{1}\right)\right\rangle+\varphi\left(g(v), g\left(u_{1}\right)\right)-\varphi\left(g\left(u_{1}\right), g\left(u_{1}\right)\right) \geq 0, & \forall v \in H, \\
\left\langle T u_{2}, g(v)-g\left(u_{2}\right)\right\rangle+\varphi\left(g(v), g\left(u_{2}\right)\right)-\varphi\left(g\left(u_{2}\right), g\left(u_{2}\right)\right) \geq 0, & \forall v \in H .
\end{array}
$$

Taking $v=u_{2}$ in (2.26) and $v=u_{1}$ in (2.27), adding the resultant and using the skewsymmetry of the bifunction $\varphi(\cdot, \cdot)$, we have

$$
\begin{aligned}
\left\langle T u_{1}-T u_{2}, g\left(u_{1}\right)-g\left(u_{2}\right)\right\rangle \leq & \varphi\left(g\left(u_{1}\right), g\left(u_{2}\right)\right)-\varphi\left(g\left(u_{1}\right), g\left(u_{1}\right)\right)-\varphi\left(g\left(u_{2}\right), g\left(u_{2}\right)\right) \\
& +\varphi\left(g\left(u_{2}\right), g\left(u_{1}\right)\right) \\
\leq & 0 .
\end{aligned}
$$

Since $T$ is strongly $g$-monotone, there exists a constant $\alpha>0$ such that

$$
\alpha\left\|g\left(u_{1}\right)-g\left(u_{2}\right)\right\|^{2} \leq\left\langle T u_{1}-T u_{2}, g\left(u_{1}\right)-g\left(u_{2}\right)\right\rangle \leq 0,
$$


which implies that $g\left(u_{1}\right)=g\left(u_{2}\right) \rightarrow u_{1}=u_{2}$, the uniqueness of the solution of $(2.1)$, since $g$ is an injective operator.

(b) Existence. We now use the auxiliary principle technique to prove the existence of a solution of (2.1). For a given $u \in H$, we consider the problem of finding a unique $w \in H$ such that

$$
\begin{aligned}
& \langle g(w), g(v)-g(w)\rangle+\rho \varphi(g(v), g(w))-\rho \varphi(g(w), g(w)) \\
& \quad \geq\langle g(u), g(v)-g(w)\rangle-\rho\langle T u, g(v)-g(w)\rangle, \quad \forall v \in H,
\end{aligned}
$$

where $\rho>0$ is a constant. The parameter $\rho$ plays a crucial part in proving that the mapping defined by the relation (2.30) is a contraction and consequently has a fixed point satisfying the original problem.

The inequality of type (2.30) is called the auxiliary variational inequality associated with problem (2.1). It is clear that the relation (2.30) defines a mapping $u \rightarrow w$. It is enough to show that the mapping $u \rightarrow w$, defined by the relation (2.30), has a fixed point belonging to $H$ satisfying the mixed quasivariational inequality $(2.1)$. Let $w_{1} \neq w_{2}$ be two solutions of (2.30) related to $u_{1}, u_{2} \in H$, respectively. It is sufficient to show that for a well-chosen $\rho>0$,

$$
\left\|w_{1}-w_{2}\right\| \leq \theta\left\|u_{1}-u_{2}\right\|
$$

with $0<\theta<1$, where $\theta$ is independent of $u_{1}$ and $u_{2}$. Taking $v=w_{2}$ (resp., $w_{1}$ ) in (2.30) related to $u_{1}$ (resp., $u_{2}$ ), adding the resultants, and using the skew-symmetry of the bifunction $\varphi(\cdot, \cdot)$, we have

$$
\left\langle g\left(w_{1}\right)-g\left(w_{2}\right), g\left(w_{1}\right)-g\left(w_{2}\right)\right\rangle \leq\left\langle g\left(u_{1}\right)-g\left(u_{2}\right)-\rho\left(T u_{1}-T u_{2}\right), g\left(w_{1}\right)-g\left(w_{2}\right)\right\rangle,
$$

from which we have

$$
\begin{aligned}
\left\|g\left(w_{1}\right)-g\left(w_{2}\right)\right\|^{2} \leq & \left\|g\left(u_{1}\right)-g\left(u_{2}\right)-\rho\left(T u_{1}-T u_{2}\right)\right\|^{2} \\
\leq & \left\|g\left(u_{1}\right)-g\left(u_{2}\right)\right\|^{2}-2 \rho\left\langle g\left(u_{1}\right)-g\left(u_{2}\right), T u_{1}-T u_{2}\right\rangle \\
& +\rho^{2}\left\|T u_{1}-T u_{2}\right\|^{2} \\
\leq & \left\|g\left(u_{1}\right)-g\left(u_{2}\right)\right\|^{2}-2 \rho \alpha\left\|g\left(u_{1}\right)-g\left(u_{2}\right)\right\|^{2}+\rho^{2} \beta^{2}\left\|g\left(u_{1}\right)-g\left(u_{2}\right)\right\|^{2} \\
\leq & \left(1-2 \rho \alpha+\rho^{2} \beta^{2}\right)\left\|g\left(u_{1}\right)-g\left(u_{2}\right)\right\|^{2},
\end{aligned}
$$

since $T$ is both a strongly monotone and Lipschitz continuous operator with constants $\alpha>0$ and $\beta>0$, respectively. Since $g$ is injective, it follows that

$$
\left\|w_{1}-w_{2}\right\| \leq \theta\left\|u_{1}-u_{2}\right\|
$$

where $\theta=\sqrt{1-2 \rho \alpha+\rho^{2} \beta^{2}}<1$ for $0<\rho<2 \alpha / \beta^{2}$, showing that the mapping defined by (2.30) has a fixed point belonging to $H$, which is the solution of $(2.1)$, the required result. 
We note that if the operator $T$ is symmetric, positive, and the bifunction $\varphi(\cdot, \cdot)$ is convex in the first argument, then the solution of the auxiliary mixed quasivariational inequality $(2.30)$ is equivalent to finding the minimum of the functional $I[w]$, where

$$
\begin{aligned}
I[w]= & \frac{1}{2}\langle g(w)-g(u), g(w)-g(u)\rangle+\rho\langle T u, g(w)-g(u)\rangle \\
& +\rho \varphi(g(u), g(w))-\rho \varphi(g(u), g(u)), \quad \forall u \in H,
\end{aligned}
$$

which is a differentiable functional associated with the inequality (2.30). This auxiliary functional can be used to construct a gap (merit) function whose stationary points solve the variational inequality (2.1). In fact, one can easily show that the mixed quasivariational inequality (2.1) is equivalent to the optimization problem. This approach is used to suggest and analyze some descent iterative methods for solving mixed quasivariational inequalities.

We also need the following condition.

Assumption 2.10. For all $u, v, w \in H$, the operator $J_{\varphi(u)}$ satisfies the condition

$$
\left\|J_{\varphi(u)} w-J_{\varphi(v)} w\right\| \leq v\|u-v\|
$$

where $v>0$ is a constant. It is shown in [12] that Assumption 2.10 is satisfied for some special cases.

Definition 2.11. A function $M: H \rightarrow R \cup\{+\infty\}$ is called a merit (gap) function for the mixed quasivariational inequalities (2.1) if and only if

(i) $M(u) \geq 0, \forall v \in H$;

(ii) $M(\bar{u})=0$ if and only if $\bar{u} \in H$ solves (2.1).

\section{Main results}

In this section, we consider some merit functions and obtain error bounds for the general mixed quasivariational inequalities (2.1) and related optimization problems. For this purpose, we need the following result, which can be proved by using Lemma 2.6.

Lemma 3.1. The general mixed quasivariational inequality (2.1) has a solution $u \in H$ if and only if $u \in H$ satisfies the relation

$$
g(u)=J_{\varphi(g(u))}[g(u)-\rho T u]
$$

where $\rho>0$ is a constant and $J_{\varphi(g(u))}=(I+\rho \partial \varphi(\cdot, g(u)))^{-1}$ is the resolvent operator.

Lemma 3.1 implies that the mixed quasivariational inequalities (2.1) are equivalent to the fixed-point problem (3.1). This alternative equivalent formulation plays an important part in suggesting and analyzing several iterative methods for solving variational inequalities. This fixed-point formulation has been used to suggest the following iterative method for problem (2.1). 
1092 Error bounds for quasivariational inequalities

We now consider the residue vector

$$
R_{\rho}(u) \equiv R(u):=g(u)-J_{\varphi(g(u))}[g(u)-\rho T u] .
$$

It is clear from Lemma 3.1 that (2.1) has a solution $u \in H$ if and only if $u \in H$ is a root of the equation

$$
R(u)=0
$$

It is known that the normal residue vector $R(u)$ defined by the relation (3.2) is a merit function for the mixed quasivariational inequality (2.1). We use this merit function to derive the global error bounds for the solution of (2.1).

Theorem 3.2. Let $\bar{u} \in H$ be a solution of (2.1) and let Assumption 2.10 hold. Let $g$ be both strongly nonexpanding and Lipschitz continuous with constants $\sigma>0$ and $\delta>0$. If the operator $T$ is both strongly $g$-monotone and $g$-Lipschitz continuous with constants $\alpha>0$ and $\beta>0$, respectively, then

$$
\frac{1}{k_{1}}\|R(u)\| \leq\|u-\bar{u}\| \leq k_{2}\|R(u)\|, \quad \forall u \in H
$$

where $k_{1}, k_{2}$ are generic constants.

Proof. Let $\bar{u} \in H$ be solution of (2.1). Then

$$
\langle T \bar{u}, g(v)-g(\bar{u})\rangle+\varphi(g(v), g(\bar{u}))-\varphi(g(\bar{u}), g(\bar{u})) \geq 0, \quad \forall v \in H .
$$

Taking $g(v)=J_{\varphi(g(u))}[g(u)-\rho T u]$ in (3.5), we have

$$
\begin{gathered}
\left\langle T \bar{u}, J_{\varphi(g(u))}[g(u)-\rho T u]-g(\bar{u})\right\rangle+\varphi\left(J_{\varphi(g(u))}[g(u)-\rho T u], g(\bar{u})\right) \\
-\varphi(g(\bar{u}), g(\bar{u})) \geq 0 .
\end{gathered}
$$

Letting $g(u)=J_{\varphi(g(u))}[g(u)-\rho T u], g(z)=g(u)-\rho T u$, and $g(v)=g(\bar{u})$ in $(2.18)$, we have

$$
\begin{aligned}
\langle\rho T u+ & \left.J_{\varphi(g(u))}[g(u)-\rho T u]-g(u), g(\bar{u})-J_{\varphi(g(u))}[g(u)-\rho T u]\right\rangle \\
& +\rho \varphi(g(\bar{u})), J_{\varphi(g(u))}[g(u)-\rho T u] \\
& -\rho \varphi\left(J_{\varphi(g(u))}[g(u)-\rho T u], J_{\varphi(g(u))}[g(u)-\rho T u]\right) \geq 0 .
\end{aligned}
$$

Adding (3.6), (3.7), and using the skew-symmetry of the bifunction $\varphi(\cdot, \cdot)$, we obtain

$$
\left\langle T \bar{u}-T u+\frac{1}{\rho}\left(g(u)-J_{\varphi(g(u))}[g(u)-\rho T u]\right), J_{\varphi(g(u))}[g(u)-\rho T u]-g(\bar{u})\right\rangle \geq 0 .
$$


Since $T$ is a strongly $g$-monotone and $g$ is nonexpanding, there exists a constant $\alpha>0$ such that

$$
\begin{aligned}
\alpha\|\bar{u}-u\|^{2} \leq & \|g(\bar{u})-g(u)\|^{2} \leq\langle T \bar{u}-T u, g(\bar{u})-g(u)\rangle \\
= & \left\langle T \bar{u}-T u, g(\bar{u})-J_{\varphi}[u-\rho T u]\right\rangle+\left\langle T \bar{u}-T u, J_{\varphi(g(u))}[g(u)-\rho T u]-g(u)\right\rangle \\
\leq & \frac{1}{\rho}\left\langle g(u)-J_{\varphi(g(u))}[g(u)-\rho T u], J_{\varphi(g(u))}[g(u)-\rho T u]-g(u)+g(u)-g(\bar{u})\right\rangle \\
& +\left\langle T \bar{u}-T u, J_{\varphi(g(u))}[g(u)-\rho T u]-g(u)\right\rangle \\
\leq & -\frac{1}{\rho}\|R(u)\|^{2}+\frac{1}{\rho}\|R(u)\|\|g(u)-g(\bar{u})\|+\|T \bar{u}-T u\|\|R(u)\| \\
\leq & \frac{1}{\rho}(1+\beta)\|R(u)\|\|g(\bar{u})-g(u)\| \\
\leq & \frac{\delta}{\rho}(1+\beta)\|R(u)\|\|\bar{u}-u\|,
\end{aligned}
$$

which implies that

$$
\|\bar{u}-u\| \leq k_{2}\|R(u)\|
$$

the right-hand inequality in (3.4) with $k_{2}=(\delta / \alpha \rho)(1+\beta)$, where $\delta>0$ is the Lipschitz constant of $g$.

Now from Assumption 2.10 and $g$-Lipschitz continuity of $T$, we have

$$
\begin{aligned}
\|R(u)\|= & \left\|g(u)-J_{\varphi(g(u))}[g(u)-\rho T u]\right\| \\
= & \left\|g(u)-g(\bar{u})+J_{\varphi(g(\bar{u}))}[g(\bar{u})-\rho T \bar{u}]-J_{\varphi(g(u))}[g(u)-\rho T u]\right\| \\
\leq & \|g(u)-g(\bar{u})\|+\left\|J_{\varphi(g(\bar{u}))}[g(\bar{u})-\rho T \bar{u}]-J_{\varphi(g(\bar{u}))}[g(u)-\rho T u]\right\| \\
& +\left\|J_{\varphi(g(\bar{u}))}[g(u)-\rho T u]-J_{\varphi(g(u))}[g(u)-\rho T u]\right\| \\
\leq & \|g(u)-g(\bar{u})\|+\nu\|g(u)-g(\bar{u})\|+\|g(u)-g(\bar{u})+\rho(T u-T \bar{u})\| \\
\leq & \{2+\nu+\rho \beta\}\|g(u)-g(\bar{u})\|=k_{1}\|u-\bar{u}\|,
\end{aligned}
$$

from which we have

$$
\frac{1}{k_{1}}\|R(u)\| \leq\|u-\bar{u}\|,
$$

the leftmost inequality in (3.4) with $k_{1}=(2+\nu+\rho \beta) \delta$, where $\delta>0$ is the Lipschitz constant of $g$. Combining (3.10) and (3.12), we obtain the required (3.4).

Letting $u=0$ in (3.4), we have

$$
\frac{1}{k_{1}}\|R(0)\| \leq\|\bar{u}\| \leq k_{2}\|R(0)\|
$$

Combining (3.4) and (3.13), we obtain the relative error bounds for any point $u \in H$. 
Theorem 3.3. Assume that all the assumptions of Theorem 3.2 hold. If $0 \neq \bar{u} \in H$ is the unique solution of (2.1), then

$$
c_{1}\left\|\frac{R(u)}{R(0)}\right\| \leq\left\|\frac{u-\bar{u}}{\bar{u}}\right\| \leq c_{2}\left\|\frac{R(u)}{R(0)}\right\|
$$

We now consider another merit function associated with problem (2.1), which can be viewed as a regularized merit function. From (2.35), we have

$$
\begin{aligned}
M_{\rho}(u):=\max _{v \in H}\{ & \langle T u, g(u)-g(v)\rangle-\varphi(g(v), g(v))+\varphi(g(u), g(v)) \\
& \left.-\frac{1}{2 \rho}\|g(u)-g(v)\|^{2}\right\}, \quad u \in H,
\end{aligned}
$$

which is called the regularized merit (gap) function associated with problem (2.1).

We note that if $\varphi(\cdot, \cdot)=\varphi(\cdot)$ is an indicator function of a closed convex set $K$ in $H$, then the merit function (3.15) reduces to the known merit function for general variational inequalities (2.4), that is,

$$
M_{\rho}(u):=\max _{v \in K}\left\{\langle T u, g(u)-g(v)\rangle-\frac{1}{2 \rho}\|g(u)-g(v)\|^{2}\right\}, \quad u \in K,
$$

which is a natural extension of a regularized merit function of Fukushima [4]. Thus it is clear that the merit function $M_{\rho}(u)$ defined by (3.15) can be viewed as a natural generalization of the regularized merit function associated with the general variational inequalities (2.4).

We note that the function $M_{\rho}(u)$ can be written as

$$
\begin{aligned}
M_{\rho}(u)= & \left\langle T u, g(u)-J_{\varphi(g(u))}[g(u)-\rho T u]\right\rangle+\varphi\left(g(u), J_{\varphi(g(u))}[g(u)-\rho T u]\right) \\
& -\varphi\left(J_{\varphi(g(u))}[g(u)-\rho T u], J_{\varphi(g(u))}[g(u)-\rho T u]\right) \\
& -\frac{1}{2 \rho}\left\|g(u)-J_{\varphi(g(u))}[g(u)-\rho T u]\right\|^{2}, \quad \forall u \in H,
\end{aligned}
$$

from which it follows that $M_{\rho}(u) \geq 0$, for all $u \in H$.

We now show that the function $M_{\rho}(u)$ defined by (3.15) is a merit function and this is the main motivation of our next result.

Theorem 3.4. For all $u \in H$,

$$
M_{\rho}(u) \geq \frac{1}{2 \rho}\|R(u)\|^{2}
$$

In particular, $M_{\rho}(u)=0$ if and only if $u \in H$ is a solution of (2.1). 
Proof. Setting $g(v)=g(u), g(u)=J_{\varphi(g(u))}[g(u)-\rho T u]$, and $g(z)=g(u)-\rho T u$ in (2.18), we have

$$
\begin{aligned}
\langle T u & \left.-\frac{1}{\rho}\left(g(u)-J_{\varphi(g(u))}[g(u)-\rho T u]\right), g(u)-J_{\varphi(g(u))}[g(u)-\rho T u]\right\rangle \\
& +\varphi\left(g(u), J_{\varphi(g(u))}[g(u)-\rho T u]\right) \\
& -\varphi\left(J_{\varphi(g(u))}[g(u)-\rho T u], J_{\varphi(g(u))}[g(u)-\rho T u]\right) \geq 0,
\end{aligned}
$$

which implies that

$$
\begin{gathered}
\langle T u, R(u)\rangle-\varphi\left(J_{\varphi(g(u))}[g(u)-\rho T u], J_{\varphi(g(u))}[g(u)-\rho T u]\right) \\
+\varphi\left(g(u), J_{\varphi(g(u))}[g(u)-\rho T u]\right) \geq \frac{1}{\rho}\|R(u)\|^{2} .
\end{gathered}
$$

Combining (3.17) and (3.20), we have

$$
\begin{aligned}
M_{\rho}(u)= & \langle T u, R(u)\rangle-\varphi\left(J_{\varphi(g(u))}[g(u)-\rho T u], J_{\varphi(g(u))}[g(u)-\rho T u]\right) \\
& +\varphi\left(g(u), J_{\varphi(g(u))}[g(u)-\rho T u]\right)-\frac{1}{2 \rho}\|R(u)\|^{2} \\
\geq & \frac{1}{2 \rho}\|R(u)\|^{2}-\frac{1}{2 \rho}\|R(u)\|^{2} \\
= & \frac{1}{2 \rho}\|R(u)\|^{2},
\end{aligned}
$$

the required result (3.18). Clearly, we have $M_{\rho}(u) \geq 0$, for all $u \in H$.

Now if $M_{\rho}(u)=0$, then clearly $R(u)=0$. Hence by Lemma 3.1, we see that $u \in H$ is a solution of (2.1). Conversely, if $u \in H$ is a solution of (2.1), then $g(u)=J_{\varphi(g(u))}[g(u)-$ $\rho T u$ ] by Lemma 3.1. Consequently, from (3.15), we see that $M_{\rho}(u)=0$, the required result.

From Theorem 3.4, we see that the function $M_{\rho}(u)$ defined by (3.15) is a merit function for the mixed quasivariational inequalities (2.1). It is known that the regularized merit function is differentiable whenever $T$ and the bifunction $\varphi(\cdot, \cdot)$ are differentiable. We now derive the error bounds without using the Lipschitz continuity of the $T$.

Theorem 3.5. Let $T$ be strongly monotone with a constant $\alpha>0$ and let the bifunction $\varphi(\cdot, \cdot)$ be a skew-symmetric function. If $g$ is strongly nonexpanding with a constant $\sigma>0$, then

$$
\|u-\bar{u}\|^{2} \leq \frac{(2 \rho)}{(2 \alpha \rho-1) \sigma M_{\rho}(u)}, \quad \forall u \in H .
$$


Proof. From (3.15) and the strong monotonicity of $T$, we have

$$
\begin{aligned}
M_{\rho}(u) \geq & \langle T u, g(u)-g(\bar{u})\rangle+\varphi(g(u), g(\bar{u}))-\varphi(g(\bar{u}), g(\bar{u}))-\frac{1}{2 \rho}\|g(u)-g(\bar{u})\|^{2} \\
\geq & \langle T \bar{u}, g(u)-g(\bar{u})\rangle+\alpha\|g(u)-g(\bar{u})\|^{2}+\varphi(g(u), g(\bar{u}))-\varphi(g(\bar{u}), g(\bar{u})) \\
& -\frac{1}{2 \rho}\|g(u)-g(\bar{u})\|^{2} .
\end{aligned}
$$

Taking $v=u$ in (3.5), we have

$$
\langle T \bar{u}, g(u)-g(\bar{u})\rangle+\varphi(g(u), g(\bar{u}))-\varphi(g(\bar{u}), g(\bar{u})) \geq 0 .
$$

From (3.23), (3.24), and using the strong nonexpansion of $g$ with constant $\sigma>0$, we have

$$
\begin{aligned}
M_{\rho}(u) & \geq \alpha\|g(u)-g(\bar{u})\|^{2}-\frac{1}{2 \rho}\|g(u)-g(\bar{u})\|^{2} \\
& =\left(\alpha-\frac{1}{2 \rho}\right) \sigma\|g(u)-g(\bar{u})\|^{2},
\end{aligned}
$$

from which the result (3.22) follows.

We consider another merit function associated with mixed quasivariational inequalities (2.1), which can be viewed as a difference of two regularized merit functions. Such a type of merit functions was introduced and studied by many authors for solving variational inequalities and complementarity problems; see $[4,20,23]$. Here we define the $D$ merit function by a formal difference of the regularized merit function defined by (3.15). To this end, we consider the function

$$
\begin{aligned}
D_{\rho, \mu}(u)=\max _{v \in H}\{ & \langle T u, g(u)-g(v)\rangle+\varphi(g(u), g(v))-\varphi(g(v), g(v)) \\
& \left.+\frac{1}{2 \rho}\|g(u)-g(v)\|^{2}-\frac{1}{2 \rho}\|g(u)-g(v)\|^{2}\right\}, \quad \forall v \in H,
\end{aligned}
$$

which is called the $D$-merit function associated with the mixed quasivariational inequalities (2.1). The $D$-merit function defined by (3.26) can be written as

$$
\begin{aligned}
D_{\rho, \mu}(u)= & \left\langle T u, J_{\varphi(g(u))}[g(u)-\mu T u]-J_{\varphi(g(u))}[g(u)-\rho T u]\right\rangle \\
& +\varphi\left(J_{\varphi(g(u))}[g(u)-\mu T u], g(u)\right)-\varphi\left(J_{\varphi(g(u))}[g(u)-\rho T u], g(u)\right) \\
& +\frac{1}{2 \mu}\left\|g(u)-J_{\varphi(g(u))}[g(u)-\mu T u]\right\|^{2}-\frac{1}{2 \rho}\left\|g(u)-J_{\varphi(g(u))}[g(u)-\rho T u]\right\|^{2} \\
= & \left\langle T u, R_{\rho}(u)-R_{\mu}(u)\right\rangle+\varphi\left(J_{\varphi(g(u))}[g(u)-\mu T u], g(u)\right) \\
& -\varphi\left(J_{\varphi(g(u))}[g(u)-\rho T u], g(u)\right)+\frac{1}{2 \mu}\left\|R_{\mu}(u)\right\|^{2} \\
& -\frac{1}{2 \rho}\left\|R_{\rho}(u)\right\|^{2}, \quad u \in H, \rho>\mu>0 .
\end{aligned}
$$


It is clear that the $D_{\rho, \mu}(u)$ is finite everywhere. We now show that the function $D_{\rho, \mu}(u)$ defined by (3.26) is indeed a merit function for the mixed quasivariational inequalities (2.1) and this is the motivation of our next result.

Theorem 3.6. For all $u \in H, \rho>\mu>0$,

$$
(\rho-\mu)\left\|R_{\rho}(u)\right\|^{2} \geq 2 \rho \mu D_{\rho, \mu}(u) \geq(\rho-\mu)\left\|R_{\mu}(u)\right\|^{2} .
$$

In particular, $D_{\rho, \mu}(u)=0$ if and only if $u \in H$ solves problem (2.1).

Proof. Taking $g(v)=J_{\varphi(g(u))}[g(u)-\mu T u], g(u)=J_{\varphi(g(u))}[g(u)-\rho T u]$, and $g(z)=g(u)-$ $\rho T u$ in (2.18), we have

$$
\begin{aligned}
\left\langle J_{\varphi(g(u))}\right. & {\left.[g(u)-\rho T u]-g(u)+\rho T u, J_{\varphi(g(u))}[g(u)-\mu T u]-J_{\varphi(g(u))}[g(u)-\rho T u]\right\rangle } \\
& +\rho \varphi\left(J_{\varphi(g(u))}[g(u)-\mu T u], J_{\varphi(g(u))}[g(u)-\rho T u]\right) \\
& -\rho \varphi\left(J_{\varphi(g(u))}[g(u)-\rho T u], J_{\varphi(g(u))}[g(u)-\rho T u]\right) \geq 0,
\end{aligned}
$$

which implies that

$$
\begin{aligned}
\left\langle T u, R_{\rho}(u)-R_{\mu}(u)\right\rangle+\varphi\left(J_{\varphi(g(u))}[g(u)-\mu T u], J_{\varphi(g(u))}[g(u)-\rho T u]\right) \\
\quad-\varphi\left(J_{\varphi(g(u))}[g(u)-\rho T u], J_{\varphi(g(u))}[g(u)-\rho T u]\right) \\
\geq \frac{1}{2 \rho}\left\langle R_{\rho}(u), R_{\rho}(u)-R_{\mu}(u)\right\rangle .
\end{aligned}
$$

From (3.27) and (3.30), we have

$$
\begin{aligned}
D_{\rho, \mu}(u) \geq & \frac{1}{2 \rho}\left\langle R_{\rho}(u), R_{\rho}(u)-R_{\mu}(u)\right\rangle+\frac{1}{2 \mu}\left\|R_{\mu}(u)\right\|^{2}-\frac{1}{2 \rho}\left\|R_{\rho}(u)\right\|^{2} \\
= & \frac{1}{2}\left(\frac{1}{\mu}-\frac{1}{\rho}\right)\left\|R_{\mu}(u)\right\|^{2}+\frac{1}{2 \rho}\left\langle R_{\rho}(u), R_{\rho}(u)-R_{\mu}(u)\right\rangle \\
& -\frac{1}{2 \rho}\left\|R_{\rho}(u)-R_{\mu}(u)\right\|^{2}-\frac{1}{2 \rho}\left\langle R_{\mu}(u), R_{\rho}(u)-R_{\mu}(u)\right\rangle \\
= & \frac{1}{2}\left(\frac{1}{\mu}-\frac{1}{\rho}\right)\left\|R_{\mu}(u)\right\|^{2}+\frac{1}{2 \rho}\left\|R_{\rho}(u)-R_{\mu}(u)\right\|^{2} \\
\geq & \frac{1}{2}\left(\frac{1}{\mu}-\frac{1}{\rho}\right)\left\|R_{\mu}(u)\right\|^{2},
\end{aligned}
$$

which implies the rightmost inequality in (3.28).

In a similar way, by taking $g(u)=J_{\varphi(g(u))}[g(u)-\mu T u], g(z)=g(u)-\mu T u$, and $g(v)=$ $J_{\varphi(g(u))}[g(u)-\rho T u]$ in $(2.18)$, we have

$$
\begin{aligned}
\left\langle J_{\varphi(g(u))}\right. & {\left.[g(u)-\mu T u]-g(u)+\mu T u, J_{\varphi(g(u))}[g(u)-\rho T u]-J_{\varphi(g(u))}[g(u)-\mu T u]\right\rangle } \\
& +\rho \varphi\left(J_{\varphi(g(u))}[g(u)-\rho T u], J_{\varphi(g(u))}[g(u)-\mu T u]\right) \\
& -\rho \varphi\left(J_{\varphi(g(u))}[g(u)-\mu T u], J_{\varphi(g(u))}[g(u)-\mu T u]\right) \geq 0,
\end{aligned}
$$


1098 Error bounds for quasivariational inequalities

which implies that

$$
\begin{aligned}
& \left\langle T u, R_{\rho}(u)-R_{\mu}(u)\right\rangle+\varphi\left(J_{\varphi(g(u))}[g(u)-\mu T u], J_{\varphi(g(u))}[g(u)-\rho T u]\right) \\
& \quad-\varphi\left(J_{\varphi(g(u))}[g(u)-\rho T u], J_{\varphi(g(u))}[g(u)-\rho T u]\right) \geq \frac{1}{2 \rho}\left\langle R_{\rho}(u), R_{\rho}(u)-R_{\mu}(u)\right\rangle .
\end{aligned}
$$

Consequently, from (3.27) and (3.33), we obtain

$$
\begin{aligned}
D_{\rho, \mu}(u) \leq & \frac{1}{\mu}\left\langle R_{\mu}(u), R_{\rho}(u)-R_{\mu}(u)\right\rangle+\frac{1}{2 \mu}\left\|R_{\mu}(u)\right\|^{2}-\frac{1}{2 \rho}\left\|R_{\rho}(u)\right\|^{2} \\
= & \frac{1}{2}\left(\frac{1}{\mu}-\frac{1}{\rho}\right)\left\|R_{\mu}(u)\right\|^{2}+\frac{1}{2 \rho}\left\langle R_{\rho}(u), R_{\rho}(u)-R_{\mu}(u)\right\rangle \\
& -\frac{1}{2 \rho}\left\|R_{\rho}(u)-R_{\mu}(u)\right\|^{2}-\frac{1}{2 \rho}\left\langle R_{\mu}(u), R_{\rho}(u)-R_{\mu}(u)\right\rangle \\
= & \frac{1}{2}\left(\frac{1}{\mu}-\frac{1}{\rho}\right)\left\|R_{\rho}(u)\right\|^{2}-\frac{1}{2} \mu\left\|R_{\rho}(u)-R_{\mu}(u)\right\|^{2} \\
\leq & \frac{1}{2}\left(\frac{1}{\mu}-\frac{1}{\rho}\right)\left\|R_{\rho}(u)\right\|^{2},
\end{aligned}
$$

which implies the leftmost inequality in (3.28).

Combining (3.31) and (3.34), we obtain (3.28), the required result.

Using essentially the technique of Theorem 3.5, we can obtain the following result.

Theorem 3.7. Let $\bar{u} \in H$ be a solution of (2.1). If the operator $T$ is strongly monotone with constant $\alpha>0$ and $g$ is strongly nonexpanding with constant $\sigma>0$, then

$$
\|u-\bar{u}\|^{2} \leq \frac{(2 \rho \mu)}{(\rho(2 \mu \alpha+1)-\mu) \sigma D_{\rho, \mu}}, \quad \forall u \in H .
$$

Proof. Let $\bar{u} \in H$ be a solution of (2.1). Then, taking $v=u$ in (3.5), we have

$$
\langle T \bar{u}, g(u)-g(\bar{u})\rangle+\varphi(g(u), g(\bar{u}))-\varphi(g(\bar{u}), g(\bar{u})) \geq 0 .
$$

Also from (3.26), (3.36), and strong monotonicity of $T$, we have

$$
\begin{aligned}
D_{\rho, \mu}(u) \geq & \langle T u, g(u)-g(\bar{u})\rangle-\varphi(g(\bar{u}), g(\bar{u}))+\varphi(g(u), g(\bar{u})) \\
& +\frac{1}{2 \mu}\|g(u)-g(\bar{u})\|^{2}-\frac{1}{2 \rho}\|g(u)-g(\bar{u})\|^{2} \\
\geq & \langle T \bar{u}, g(u)-g(\bar{u})\rangle-\varphi(g(\bar{u}), g(\bar{u}))+\varphi(g(u), g(\bar{u})) \\
& +\alpha\|g(u)-g(\bar{u})\|^{2}+\frac{1}{2 \mu}\|g(u)-g(\bar{u})\|^{2}-\frac{1}{2 \rho}\|g(u)-g(\bar{u})\|^{2} \\
\geq & \sigma\left(\alpha+\frac{1}{2 \mu}-\frac{1}{2 \rho}\right)\|u-\bar{u}\|^{2},
\end{aligned}
$$

from which the required result (3.35) follows, where $\sigma>0$ is a strongly expansivoty constant of $g$. 


\section{Acknowledgement}

The author expresses his sincere appreciation to the referee for valuable comments and constructive suggestions.

\section{References}

[1] C. Baiocchi and A. Capelo, Variational and Quasivariational Inequalities, A Wiley-Interscience Publication, John Wiley \& Sons, New York, 1984.

[2] H. Brézis, Opérateurs Maximaux Monotones et Semi-Groupes de Contractions dans les Espaces de Hilbert, North-Holland Mathematics Studies, No. 5, North-Holland Publishing, Amsterdam, 1973.

[3] J. Crank, Free and Moving Boundary Problems, Oxford Science Publications, The Clarendon Press Oxford University Press, New York, 1984.

[4] M. Fukushima, Equivalent differentiable optimization problems and descent methods for asymmetric variational inequality problems, Math. Program. 53 (1992), no. 1, Ser. A, 99-110.

[5] F. Giannessi and A. Maugeri (eds.), Variational Inequalities and Network Equilibrium Problems, Plenum Press, New York, 1995, Proceedings of the conference held in Erice, June 19-25, 1994.

[6] F. Giannessi, A. Maugeri, and P. M. Pardalos (eds.), Equilibrium Problems: Nonsmooth Optimization and Variational Inequality Methods, Nonconvex Optimization and its Applications, vol. 58, Kluwer Academic Publishers, Dordrecht, 2001.

[7] R. Glowinski, J.-L. Lions, and R. Trémolières, Numerical Analysis of Variational Inequalities, Studies in Mathematics and its Applications, vol. 8, North-Holland Publishing, Amsterdam, 1981.

[8] N. Kikuchi and J. T. Oden, Contact Problems in Elasticity: A Study of Variational Inequalities and Finite Element Methods, SIAM Studies in Applied Mathematics, vol. 8, Society for Industrial and Applied Mathematics (SIAM), Pennsylvania, 1988.

[9] D. Kinderlehrer and G. Stampacchia, An Introduction to Variational Inequalities and Their Applications, Classics in Applied Mathematics, vol. 31, Society for Industrial and Applied Mathematics (SIAM), Pennsylvania, 2000.

[10] M. A. Noor, General variational inequalities, Appl. Math. Lett. 1 (1988), no. 2, 119-122.

[11] Nonlinear variational inequalities in elastostatics, Internat. J. Engrg. Sci. 26 (1988), no. $10,1043-1051$.

[12] - Generalized quasi variational inequalities and implicit Wiener-Hopf equations, Optimization 45 (1999), no. 1-4, 197-222.

[13] - Set-valued mixed quasi-variational inequalities and implicit resolvent equations, Math. Comput. Modelling 29 (1999), no. 5, 1-11.

[14] - Iterative methods for general mixed quasivariational inequalities, J. Optim. Theory Appl. 119 (2003), no. 1, 123-136.

[15] _ Mixed quasi variational inequalities, Appl. Math. Comput. 146 (2003), no. 2-3, 553578.

[16] - Some developments in general variational inequalities, Appl. Math. Comput. 152 (2004), no. 1, 199-277.

[17] M. A. Noor and K. I. Noor, On general mixed quasivariational inequalities, J. Optim. Theory Appl. 120 (2004), no. 3, 579-599.

[18] M. A. Noor, K. I. Noor, and T. M. Rassias, Some aspects of variational inequalities, J. Comput. Appl. Math. 47 (1993), no. 3, 285-312.

[19] M. Patriksson, Nonlinear Programming and Variational Inequality Problems. A Unified Approach, Applied Optimization, vol. 23, Kluwer Academic Publishers, Dordrecht, 1999. 


\section{Error bounds for quasivariational inequalities}

[20] J.-M. Peng, Equivalence of variational inequality problems to unconstrained minimization, Math. Program. 78 (1997), no. 3, Ser. A, 347-355.

[21] J.-M. Peng and M. Fukushima, A hybrid Newton method for solving the variational inequality problem via the D-gap function, Math. Program. 86 (1999), no. 2, Ser. A, 367-386.

[22] B. Qu, C. Y. Wang, and J. Z. Zhang, Convergence and error bound of a method for solving variational inequality problems via the generalized D-gap function, J. Optim. Theory Appl. 119 (2003), no. 3, 535-552.

[23] M. V. Solodov, Merit functions and error bounds for generalized variational inequalities, J. Math. Anal. Appl. 287 (2003), no. 2, 405-414.

[24] M. V. Solodov and P. Tseng, Some methods based on the D-gap function for solving monotone variational inequalities, Comput. Optim. Appl. 17 (2000), no. 2-3, 255-277.

[25] G. Stampacchia, Formes bilinéaires coercitives sur les ensembles convexes, C. R. Acad. Sci. Paris 258 (1964), 4413-4416 (French).

[26] N. H. Xiu and J. Z. Zhang, Global projection-type error bounds for general variational inequalities, J. Optim. Theory Appl. 112 (2002), no. 1, 213-228.

Muhammad Aslam Noor: Mathematics Department, COMSATS Institute of Information Technology, University Road, Abbottabad, Islambad, Pakistan

E-mail address: noormaslam@hotmail.com

Khalida Inayat Noor: Mathematics Department, COMSATS Institute of Information Technology, University Road, Abbottabad, Islambad, Pakistan

E-mail address: khalidanoor@hotmail.com 


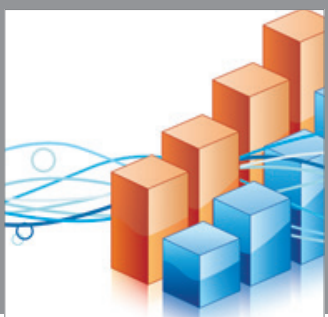

Advances in

Operations Research

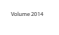

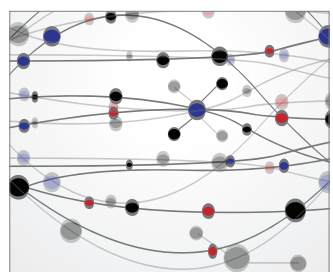

\section{The Scientific} World Journal
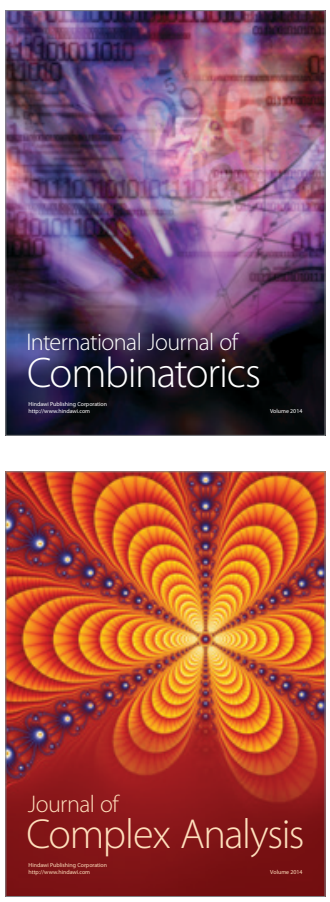

International Journal of

Mathematics and

Mathematical

Sciences
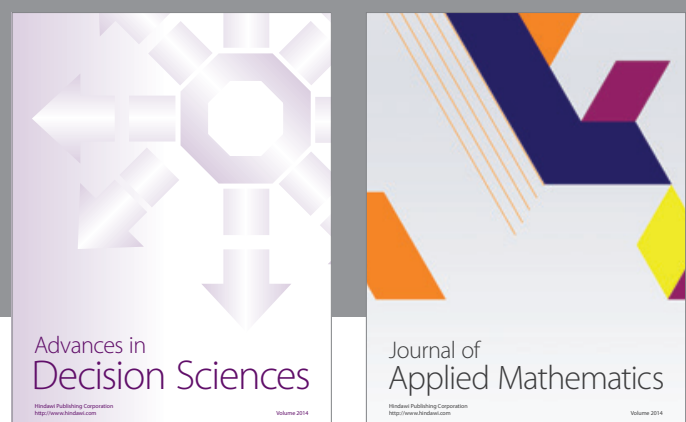

Journal of

Applied Mathematics
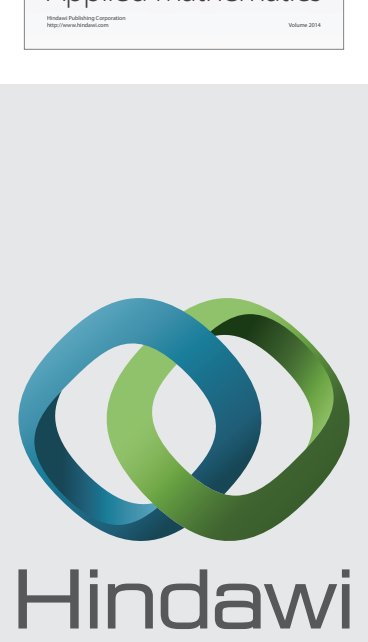

Submit your manuscripts at http://www.hindawi.com
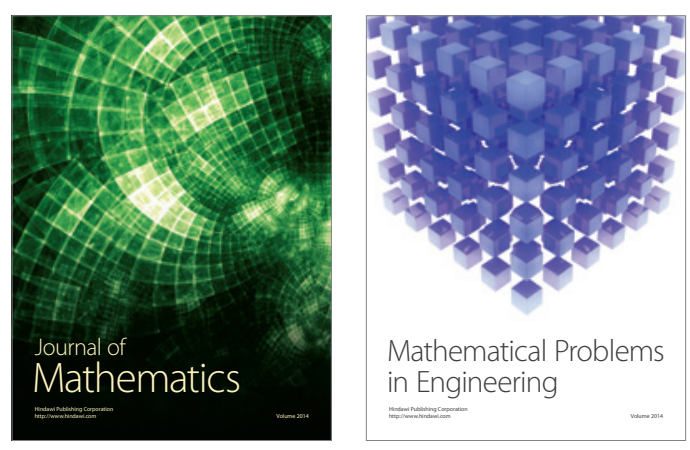

Mathematical Problems in Engineering
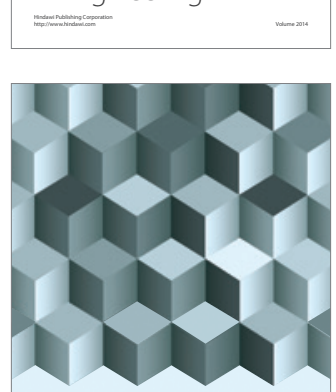

Journal of

Function Spaces
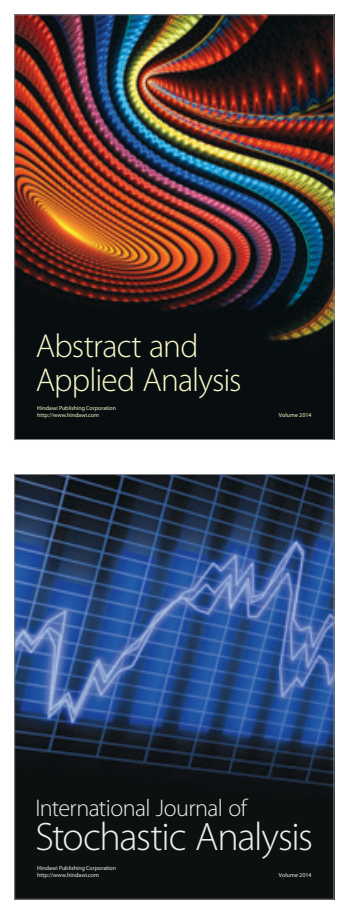

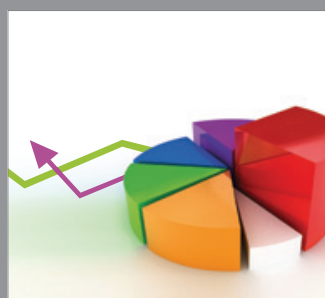

ournal of

Probability and Statistics

Promensencen
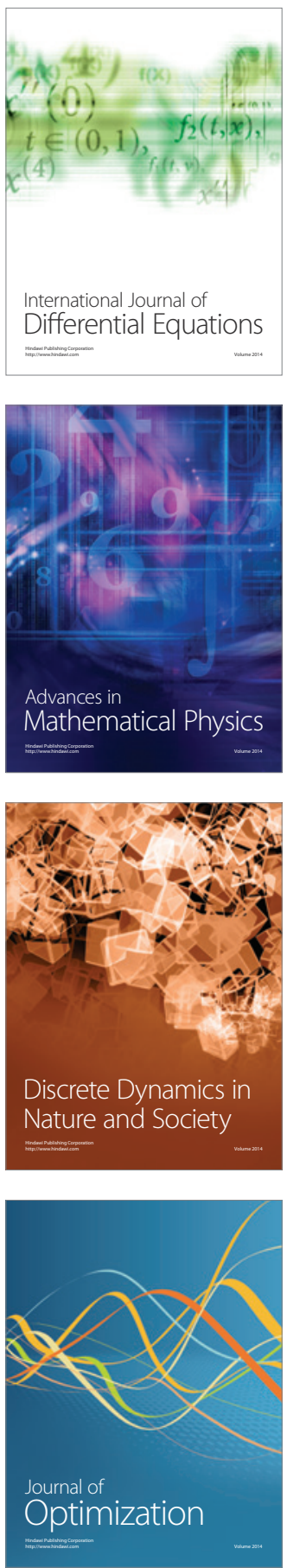